\title{
Aplicativos móveis desenvolvidos para Educação em Saúde no mundo: revisão
}

\section{integrativa da literatura}

\author{
Mobile applications developed for Health Education in the world: integrative literature review \\ Aplicaciones Móviles desarrolladas para la Educación Sanitaria en el mundo: revisión integrativa
}

de la literatura

Recebido: 02/10/2021 | Revisado: 09/10/2021 | Aceito: 13/10/2021 | Publicado: 16/10/2021

Geyse do Espírito Santo Rezende

ORCID: https://orcid.org/0000-0002-2570-0543 Universidade Federal de Sergipe, Brasil E-mail: geyserezende@gmail.com

André Luiz França Alves

ORCID: https://orcid.org/0000-0003-2491-7633 Universidade Federal de Sergipe, Brasil E-mail: andrelfalves@outlook.com

Andreia Ferreira de Aragão Rabelo

ORCID: https://orcid.org/0000-0003-1650-1259 Universidade Federal de Sergipe, Brasil E-mail: andreia_far@yahoo.com.br

\begin{abstract}
Resumo
O objetivo deste estudo é verificar o que foi desenvolvido na literatura científica mundial em aplicativos móveis para Educação em Saúde voltado para o público adulto, nos últimos 10 anos. Trata-se de uma revisão integrativa da literatura, tendo como amostra final 28 artigos; os resultados foram analisados com relação ao ano, local do estudo, categoria do aplicativo, desfecho e desdobramento. Também foi feita a busca por habilidades do produto que levassem a maior interação com o público alvo e com a mudança no comportamento do usuário frente as suas condições de saúde; assim pode ser visto que dentre os 28 artigos selecionados, 19 trabalhos apresentaram pontos de melhoria a serem implementados na respectiva tecnologia, enquanto 7 não apresentam. Desta forma, foi concluído que o investimento no desenvolvimento de aplicativos com esse objetivo é promissor ao ser considerado o engajamento que as tecnologias têm nos dias atuais, tendo grande potencial inovador.
\end{abstract}

Palavras-chave: Aplicativos; Smatphone; Educação; Saúde.

\begin{abstract}
The aim of this study is to verify what has been developed in the world scientific literature in mobile applications for health education aimed at the adult audience, in the last 10 years. This is an integrative literature review, with a final sample of 28 articles; Results were analyzed by year, study location, application category, outcome, and outcome. A search was also made for product skills that would lead to greater interaction with the target audience and with a change in the user's behavior towards their health conditions; thus, it can be seen that among the 28 selected articles, 19 works presented points of improvement to be implemented in the respective technology, while 7 did not. Thus, it was concluded that the investment in application development with this objective is promising when considering the engagement that technologies have nowadays, with great innovative potential.
\end{abstract}

Keywords: Apps; Smartphone; Health; Education; Health.

\section{Resumen}

El objetivo de este estudio es verificar lo desarrollado en la literatura científica mundial en aplicaciones móviles de educación para la salud dirigidas a la audiencia adulta, en los últimos 10 años. Se trata de una revisión bibliográfica integradora, con una muestra final de 28 artículos; Los resultados se analizaron por año, ubicación del estudio, categoría de aplicación, resultado y resultado. También se realizó una búsqueda de habilidades de producto que conducirían a una mayor interacción con el público objetivo y a un cambio en el comportamiento del usuario hacia sus condiciones de salud; así, se puede observar que entre los 28 artículos seleccionados, 19 trabajos presentaron puntos de mejora para ser implementados en la tecnología respectiva, mientras que 7 no. Así, se concluyó que la inversión en desarrollo de aplicaciones con este objetivo es prometedora si se considera el compromiso que tienen las tecnologías en la actualidad, con gran potencial innovador

Palabras clave: Aplicaciones; Teléfono inteligente; Educación; Salud. 


\section{Introdução}

As tecnologias têm um impacto significativo nos seres humanos e na sociedade. Eles transformam nossas maneiras de pensar, sentir e agir, assim como a forma como nos comunicamos e obtemos conhecimento (Kenski, 2008).

As maneiras pelas quais aprendemos e ensinamos foram influenciadas por avanços tecnológicos (Martin, et al.,2011) e tecnologias usadas para fins educacionais tem crescido progressivamente (Berrett, Murphy, \& Sullivan, 2012; Inan e Lowther, 2010). Atualmente, a educação on-line está rompendo barreiras geográficas e fornecendo oportunidades de aprendizagem onipresente (Shukor, et al., 2014), abraçados por instituições de ensino em todo o mundo. Tem potencial para cunhar processos de aprendizagem mais centrada no aluno (Dwivedi, et al., 2019) e para promover a aprendizagem cognitiva, aspectos metacognitivos, afetivo-motivacionais e sociais (Johnson \& Johnson, 2009).

A Educação em Saúde é uma vertente da atividade profissional que visa desenvolver o pensamento crítico fundamentado em um saber científico; tem potencial para gerar mudanças significativas no comportamento das pessoas de forma a produzir melhora na condição de saúde a partir do empoderamento do sujeito sobre sua própria saúde (Fontana, et al., 2020).

Devido ao desenvolvimento de tecnologias de computador e multimídia, muitos estudiosos tentaram empregar conteúdo de aprendizado digital para melhorar a motivação de aprendizagem dos alunos. Muitos estudos examinaram a eficácia da aprendizagem aprimorada por tecnologia e relataram seus benefícios (Abdulla, 2012; Chow, Herold, Choo \& Chan, 2012; Pivec, 2007).

Por ter se tornado tão popular, os smartphones são uma porta de acesso para que haja o investimento no desenvolvimento de aplicativos; estes, quando são utilizados em consonância com medidas de saúde, podem potencializar o tratamento e aumentar o ganho de conhecimento (Heffernan, et al., 2016; Oliveira, et al., 2016).

Nos últimos anos, houve um grande aumento na criação de aplicativos móveis voltados para a área da saúde, sendo estes instrumentos muito úteis no contexto de promoção, informação e Educação em Saúde; com caráter inovador, os aplicativos vêm como um diferencial na atualidade do contexto da saúde (Oliveira e Alencar, 2017).

Desta forma, o presente artigo busca extrair da literatura científica mundial o que existe de aplicativos móveis desenvolvidos para Educação em Saúde voltado para o público adulto, nos últimos 10 anos.

\section{Metodologia}

Trata-se de uma Revisão Integrativa, um método de pesquisa que permite a síntese de vários estudos publicados e que possibilita trazer conclusões gerais a respeito de uma área de estudo bem definida. A Revisão integrativa tem etapas bem definidas, que são: definição da pergunta norteadora, coleta de dados, avaliação dos dados, análise e interpretação de dados e a apresentação da revisão (Mendes, et al, 2008).

Por se tratar de uma revisão integrativa da literatura, não foi necessária a aprovação do estudo pelo comitê de ética em pesquisa, segundo a Resolução 466/12 do Ministério da Saúde.

A questão norteadora desse estudo é: o que foi desenvolvido no mundo, na área de Educação em Saúde, relacionado a aplicativos móveis?

Com esse foco, no dia 30 de maio de 2020 foram realizadas buscas em cinco (05) bases de dados: Excerpta Medica dataBASE (Embase), Scientific Electronic Library Online (SciELO), Scopus, Web of Science e ScienceDirect, através da seguinte string de busca: (App AND health AND smartphone).

Os critérios de inclusão de artigos utilizados foram os seguintes: publicações disponíveis de forma completa e gratuita nas bases de dados, escritos em Inglês, Português e/ou Espanhol, publicados no período compreendido entre 2010 e 2020. 
Os critérios de exclusão foram: artigos que, após leitura de título e resumo, não foi possível identificar uma afinidade com a temática proposta; artigos de revisão sistemática, editoriais, meta-análise e carta ao editor; artigo de comparação entre aplicativos ou que tivessem como objetivo realizar um apanhado de uma variedade de aplicativos

O processo de seleção dos artigos foi realizado com a aplicação dos critérios de elegibilidade e está apresentado na Figura 1:

Figura 1 - Síntese do processo de seleção dos artigos para a revisão integrativa.

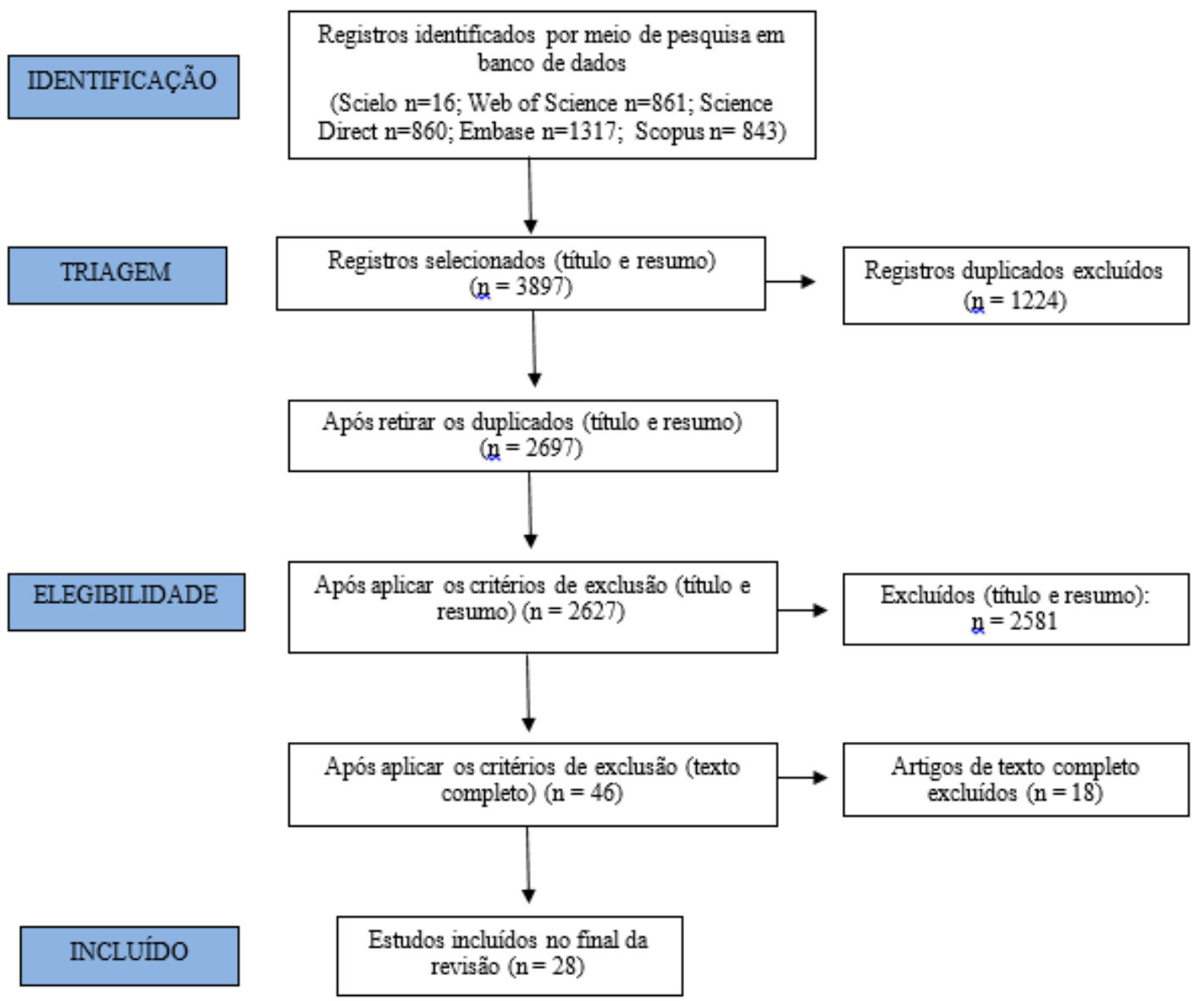

Fonte: Autores (2020).

Após as buscas nas bases de dados, foram selecionados o total de 3897 artigos, dos quais foram excluídos inicialmente 1224 por se tratarem de textos duplicados, restando 2673 artigos para serem analisados a partir da leitura do título e resumo; após esta leitura, foram eliminados 2627 artigos que não se enquadravam nos critérios do estudo e foram selecionados artigos que discorressem sobre aplicativos voltados para a Educação em Saúde, sendo estes o total de 46 artigos. Após essa pré-seleção dos artigos, foi feita uma leitura minuciosa das publicações para definir a inclusão e exclusão desses artigos de acordo com os critérios estabelecidos, tendo-se excluídos mais 17 artigos, restando então 28 artigos para a análise completa. Após a escolha definitiva dos estudos a serem analisados na revisão, realizou-se a coleta de informações quantitativas (local de publicação, autor, ano de publicação, periódico, público alvo e base de dados) e informações qualitativas como título, objetivos, desfecho e desdobramentos. 


\section{Resultados}

Após buscas realizadas nas bases de dados, foram encontrados 28 artigos que respondiam à questão norteadora do estudo. Os trabalhos selecionados foram sumarizados e classificados de acordo com os seguintes critérios quantitativos: país de publicação, ano de publicação, categoria de interesse do aplicativo e periódico onde o artigo foi publicado.

Em relação ao país de publicação dos artigos encontrados, seis foram desenvolvidos no Reino Unido (Inglaterra e Irlanda) quatro nos EUA, quatro na Arábia Saudita, dois no Brasil e dois na Austrália. Os demais se distribuíram, com um artigo cada, em países da Europa (Espanha, Suécia, Dinamarca, Itália, Holanda e Portugal).

Quanto ao ano de publicação dos artigos, as publicações datam a partir do ano de 2014. A maioria foi publicado em 2019 (dez artigos), seguidos de oito em 2018 e quatro, em 2016. Os demais artigos foram publicados em 2020 (03 artigos), 2015 (03 artigos), 2017 (01), 2014 (01). Todos os artigos selecionados foram escritos e publicados no idioma Inglês.

Esses dados estão melhor descritos no Quadro 1. 
Research, Society and Development, v. 10, n. 13, e352101321334, 2021

(CC BY 4.0) | ISSN 2525-3409 | DOI: http://dx.doi.org/10.33448/rsd-v10i13.21334

Quadro 1 - Dados gerais dos artigos selecionados.

\begin{tabular}{|c|c|c|c|c|c|}
\hline Base & $\begin{array}{c}\text { Referência / } \\
\text { Local }\end{array}$ & Aplicativo & Objetivos & Desfechos & Desdobramentos \\
\hline Embase & $\begin{array}{l}\text { (Ragavan, } \\
\text { Ferre e Bair- } \\
\text { Merritt, } \\
\text { 2020) } \\
\text { EUA }\end{array}$ & Thrive & $\begin{array}{c}\text { Coletar feedbacks a respeito do aplicativo } \\
\text { Thrive }\end{array}$ & A revisão, o teste para disseminar o Thrive & $\begin{array}{l}\text { Usuários adicionem seu próprio conteúdo e } \\
\text { forneçam mecanismos de apoio social. }\end{array}$ \\
\hline Embase & $\begin{array}{l}\text { (Borgen, et } \\
\text { al., 2019) } \\
\text { Noruega }\end{array}$ & Grávida + & Avaliar o efeito do aplicativo Grávida + & O aplicativo Grávida + não teve efeito & $\begin{array}{l}\text { Pesquisas futuras devem investigar quais recursos } \\
\text { específicos dos aplicativos podem melhorar }\end{array}$ \\
\hline Embase & $\begin{array}{l}\text { (Doyle, et } \\
\text { al., 2019) } \\
\text { Irlanda }\end{array}$ & MiKidney & Avaliar o aplicativo MiKidney & $\begin{array}{l}\text { Tem potencial para capacitar e motivar os pacientes a } \\
\text { entender e autogerenciar sua condição. }\end{array}$ & $\begin{array}{l}\text { Precisa de mais desenvolvimento, incluindo } \\
\text { gravação e rastreamento de dieta e ingestão de } \\
\text { líquidos, pressão arterial e aplicativo uso. }\end{array}$ \\
\hline Embase & $\begin{array}{l}\text { (Dale, et al., } \\
\text { 2019) } \\
\text { Inglaterra }\end{array}$ & $\begin{array}{c}\text { Carrot } \\
\text { Rewards }\end{array}$ & $\begin{array}{c}\text { Avaliar a aceitação do questionário da } \\
\text { Campanha contra a Gripe Cenoura, as } \\
\text { notificações móveis "push", e incentivos ao } \\
\text { ponto de fidelidade. }\end{array}$ & $\begin{array}{c}\text { Usou um novo método de entrega para educar a } \\
\text { população em geral e mostrou a geolocalização poderia } \\
\text { ser usada para facilitar a captação da vacina contra } \\
\text { influenza. }\end{array}$ & $\begin{array}{l}\text { As iterações futuras podem adaptar o conteúdo } \\
\text { que estão mais em risco e deve considerar } \\
\text { métodos de avaliação mais robustos }\end{array}$ \\
\hline Embase & $\begin{array}{l}\text { (Requena, et } \\
\text { al., 2019) } \\
\text { Espanha }\end{array}$ & Farmalarm & $\begin{array}{l}\text { Comparar, o grau de controle dos seguintes } \\
\text { fatores de risco vasculares entre ambos os } \\
\text { grupos: hipertensão, diabetes mellitus, } \\
\text { colesterol e tabagismo }\end{array}$ & $\begin{array}{l}\text { O uso de aplicativos móveis para monitorar a } \\
\text { conformidade com os medicamentos e aumentar a } \\
\text { conscientização sobre o AVC é viável }\end{array}$ & N/A \\
\hline Embase & $\begin{array}{l}\text { (Abrams, et } \\
\text { al., 2019) } \\
\text { EUA }\end{array}$ & The Bridge & $\begin{array}{l}\text { diminuir complicações que levam a readmissões } \\
\text { hospitalares não planejadas,bem como melhorar } \\
\text { a qualidade de vida e suporte do paciente }\end{array}$ & $\begin{array}{l}\text { Os melhores resultados foram para pacientes com } \\
\text { diabetes, hipertensão,asma, gravidez e tabagismo, } \\
\text { espera-se ser um complemento para cumprir planos para } \\
\text { esses paciente }\end{array}$ & $\begin{array}{c}\text { Existem possíveis barreiras ao uso bem-sucedido } \\
\text { do Aplicativo móvel. }\end{array}$ \\
\hline Embase & $\begin{array}{c}\text { (Rahim } \\
\text { Shilbayeh, } \\
\text { et al., 2019) } \\
\text { Arábia } \\
\text { Saudita }\end{array}$ & CATA mApp & $\begin{array}{c}\text { Explorar o impacto de um aplicativo móvel } \\
\text { desenvolvido }\end{array}$ & $\begin{array}{c}\text { Tem um vasto potencial para melhorar o conhecimento } \\
\text { dos pacientes; no entanto, requer investigação em } \\
\text { estudos prospectivos de longo prazo com maior } \\
\text { frequência }\end{array}$ & $\begin{array}{l}\text { Nossos estudos futuros abordarão aprimoramento } \\
\text { e desenvolvimento do mApp }\end{array}$ \\
\hline Embase & $\begin{array}{l}\text { (Yuan e } \\
\text { Marques, } \\
\text { 2018) }\end{array}$ & ProFibro & $\begin{array}{c}\text { Desenvolver um app para promover o } \\
\text { autocuidado como recurso complementar de } \\
\text { Saúde Móvel à fisioterapia no tratamento da } \\
\text { fibromialgia. }\end{array}$ & $\begin{array}{l}\text { Fisioterapeutas podem recomendam o uso deste } \\
\text { aplicativo a seus pacientes como recurso complementar } \\
\text { para promover o autocuidado na fibromialgia }\end{array}$ & $\begin{array}{l}\text { realizar um estudo randomizado para avaliar a } \\
\text { eficácia do app ProFibro nos pro-movimento de } \\
\text { autocuidado e melhora dos sintomas e qualidade } \\
\text { de vida }\end{array}$ \\
\hline
\end{tabular}


Research, Society and Development, v. 10, n. 13, e352101321334, 2021

(CC BY 4.0) | ISSN 2525-3409 | DOI: http://dx.doi.org/10.33448/rsd-v10i13.21334

\begin{tabular}{|c|c|c|c|c|c|}
\hline & Brasil & & & & \\
\hline Embase & $\begin{array}{l}\text { (Bonn, et al., } \\
\text { 2018) } \\
\text { Suécia }\end{array}$ & DiaCert & $\begin{array}{l}\text { Descrever o desenho do estudo e metodologia } \\
\text { do estudo DiaCert. }\end{array}$ & $\begin{array}{c}\text { É uma nova solução de compartilhamento de dados } \\
\text { incluindo um aplicativo de smartphone para os pacientes } \\
\text { e um aplicativo digital plataforma para o pessoal de } \\
\text { saúde }\end{array}$ & N/A \\
\hline Embase & $\begin{array}{l}\text { (Shalan, et } \\
\text { al., 2018) } \\
\text { Inglaterra } \\
\end{array}$ & YORwalK & $\begin{array}{l}\text { Desenvolver um protótipo desse aplicativo, para } \\
\text { promover exercícios e rastrear mudanças na } \\
\text { capacidade da população caminhar. }\end{array}$ & $\begin{array}{c}\text { O aplicativo está disponível apenas para telefones } \\
\text { Android modernos e isso é uma fraqueza nesta versão; } \\
\text { deve ser capaz de se conectar a dispositivos de fitness } \\
\text { vestíveis }\end{array}$ & $\begin{array}{l}\text { buscar feedback dos pacientes por meio de um } \\
\text { fórum de pacientes. }\end{array}$ \\
\hline Embase & $\begin{array}{l}\text { (Brett, } \\
\text { Boulton e } \\
\text { Watson, } \\
\text { 2018) } \\
\text { Inglaterra }\end{array}$ & Yagmap & $\begin{array}{l}\text { Explorar, desenvolver e pilotar um aplicativo de } \\
\text { saúde eletrônica centrado no paciente para } \\
\text { apoiar e motivar as mulheres que tomam AET. }\end{array}$ & $\begin{array}{c}\text { Os fundos limitados impediram o desenvolvimento de } \\
\text { um aplicativo de saúde eletrônica mais tecnicamente } \\
\text { avançado para as mulheres após o tratamento para o } \\
\text { câncer de mama }\end{array}$ & $\begin{array}{c}\text { É necessário um maior desenvolvimento e } \\
\text { avaliação do aplicativo para determinar se ele } \\
\text { apoia as mulheres e melhora a adesão à AET } \\
\text { após o tratamento }\end{array}$ \\
\hline Embase & $\begin{array}{l}\text { (Nørgaard, } \\
\text { et al., 2017) } \\
\text { Dinamarca }\end{array}$ & $\begin{array}{l}\text { Pregnant with } \\
\text { diabetes }\end{array}$ & $\begin{array}{l}\text { Avaliar a conscientização e o uso do aplicativo } \\
\text { (aplicativo informativo projetado para mulheres } \\
\text { grávidas com diabetes). }\end{array}$ & $\begin{array}{l}\text { Informações de pacientes chegam aos pacientes e podem } \\
\text { contribuir para um melhor planejamento e resultado da } \\
\text { gravidez em mulheres com diabetes }\end{array}$ & N/A \\
\hline Embase & $\begin{array}{l}\text { (Bender, } \\
\text { Martinez e } \\
\text { Kennedy, } \\
\text { 2016) } \\
\text { EUA }\end{array}$ & VELT & $\begin{array}{l}\text { Descrever a promoção práticas e experiências } \\
\text { atuais des-seminários de Educação em Saúde }\end{array}$ & $\begin{array}{l}\text { Tem o potencial para fornecer um modelo que possa } \\
\text { melhorar os promover a mudança de comportamento no } \\
\text { estilo de vida saudável }\end{array}$ & $\begin{array}{l}\text { são necessárias avaliar a viabilidade e eficácia } \\
\text { potencial de uma cultura aplicativo Latly VELT } \\
\text { mHealth apropriado para promover PA }\end{array}$ \\
\hline Embase & $\begin{array}{l}\text { (Panatto, et } \\
\text { al., 2016) } \\
\text { Itália }\end{array}$ & $\begin{array}{l}\text { Pneumo } \\
\text { Rischio }\end{array}$ & $\begin{array}{l}\text { Desenvolver e implementar um aplicativo } \\
\text { móvel fácil de usar para aumentar o } \\
\text { conhecimento dos leigos, a conscientização e a } \\
\text { percepção de risco pessoal de DPI }\end{array}$ & $\begin{array}{l}\text { Tem o potencial de melhorar a conscientização } \\
\text { relativamente baixa da DPI entre os profissionais de } \\
\text { saúde, o que pode reduzir os níveis de vacinação entre } \\
\text { seus pacientes. }\end{array}$ & $\begin{array}{c}\text { Avaliar a satisfação do usuário, identificar } \\
\text { estratégias de marketing social para aumentar a } \\
\text { exposição da comunidade (especialmente entre } \\
\text { homens e idosos) }\end{array}$ \\
\hline Embase & $\begin{array}{l}\text { (Athilingam, } \\
\text { et al., 2016) } \\
\text { EUA }\end{array}$ & $\begin{array}{l}\text { Heart mapp } \\
\text { education }\end{array}$ & $\begin{array}{c}\text { Incorporar a educação interativa para } \\
\text { insuficiência cardíaca em uma plataforma } \\
\text { móvel }\end{array}$ & $\begin{array}{l}\text { As atividades educacionais envolvem tomadas de } \\
\text { decisão compartilhadas para melhorar práticas de } \\
\text { autocuidado. Em um ambiente de saúde cada vez mais } \\
\text { ocupado, profissionais e enfermeiros podem ajudar } \\
\text { melhor o paciente explorar o aplicativo em seu próprio } \\
\text { celular para obter relevância informações de saúde em } \\
\text { seu próprio ritmo em casa sem a medo de perder a } \\
\text { informação impressa. Para médicos e enfermeiros, a } \\
\text { saúde móvel pode levar a demandas reduzidas em seu }\end{array}$ & $\begin{array}{l}\text { A educação na alta é um componente vital para } \\
\text { melhorar resultados. Incorporando a Educação } \\
\text { em Saúde em uma plataforma móvel proposto } \\
\text { para melhorar o envolvimento do paciente, } \\
\text { facilitar comunicação, ajudar a superar os } \\
\text { desafios da saúde, atender a tomada de decisão e } \\
\text { melhorar o gerenciamento de doenças resultados. } \\
\text { Acreditamos que, à medida que o tempo avança e } \\
\text { a familiaridade com a tecnologia se torna uma }\end{array}$ \\
\hline
\end{tabular}




\begin{tabular}{|c|c|c|c|c|c|}
\hline & & & & $\begin{array}{l}\text { tempo e permitir que eles se concentrem na arte da } \\
\text { medicina e } \\
\text { Ainda há muito a ser feito para conduzir essa } \\
\text { transformação. } \\
\text { A necessidade mais crítica é um ensaio clínico para } \\
\text { fornecer um roteiro para implementação que confirme } \\
\text { seus benefícios para os consumidores, médicos, } \\
\text { enfermeiros e contribuintes. }\end{array}$ & $\begin{array}{l}\text { segunda natureza, os pacientes experimentam } \\
\text { ansiedades decrescentes associadas à educação } \\
\text { aplicativos em uma plataforma móvel. } \\
\text { Planejamos testar o aplicativo em um ensaio } \\
\text { clínico por sua eficácia na melhoria dos } \\
\text { resultados da IC em um estudo controlado } \\
\text { randomizado para dois grupos, comparado com a } \\
\text { atual versão em papel padrão de material } \\
\text { educacional. }\end{array}$ \\
\hline Embase & $\begin{array}{l}\text { (Glynn, et } \\
\text { al., 2014) } \\
\text { Irlanda }\end{array}$ & $\begin{array}{c}\text { Accupedo-pro } \\
\text { pedometer }\end{array}$ & $\begin{array}{c}\text { Avaliar a eficácia de um aplicativo para } \\
\text { smartphone no aumento da atividade física na } \\
\text { atenção primária. }\end{array}$ & $\begin{array}{c}\text { Tais tecnologias baratas, acessíveis e fáceis de usar } \\
\text { devem ser consideradas um componente de qualquer } \\
\text { intervenção futura para promover a atividade física na } \\
\text { atenção primária. }\end{array}$ & $\begin{array}{c}\text { Um aplicativo simples para smartphone } \\
\text { aumentou significativamente a atividade física ao } \\
\text { longo de } 8 \text { semanas em uma população de } \\
\text { atenção primária. }\end{array}$ \\
\hline Scopus & $\begin{array}{l}\text { (Stauber, et } \\
\text { al., 2020) } \\
\text { Arábia } \\
\text { Saudita }\end{array}$ & Recover-E & $\begin{array}{c}\text { Objetivo de desenvolver as habilidades } \\
\text { necessárias de autogestão para facilitar a } \\
\text { recuperação e garantir o sucesso a longo prazo. } \\
\text { Desenvolvemos um aplicativo móvel } \\
\text { (RECOVER-E) para iOS e Android } \\
\text { smartphones que fornecem informações } \\
\text { importantes sobre a fase pré-operatória, cirurgia } \\
\text { e recuperação }\end{array}$ & $\begin{array}{c}\text { Despecho primário: será a função física } \\
\text { autorreferidamedido nas subescalas de atividades da } \\
\text { vida diária (AVD) da lesão no joelho e no escore de } \\
\text { resultado da osteoartrite(KOOS) eo escore de resultado } \\
\text { de deficiência de quadril e osteoartrite (HOOS) para } \\
\text { pacientes com joelho e quadrilosteoartrite, } \\
\text { respectivamente.Desfecho secundário: incluem as } \\
\text { subescalas de dor, sintomas, função no esporte e } \\
\text { recreação e joelho / quadril.qualidade de vida } \\
\text { relacionada ao HOOS e KOOS, ansiedade pré- } \\
\text { operatório }\end{array}$ & $\begin{array}{l}\text { O estudo deve fornecer evidências que apóiem o } \\
\text { uso da saúde móvel para facilitar a recuperação e } \\
\text { abrir novas possibilidades para atendimento ao } \\
\text { paciente em substituição do joelho ou quadril }\end{array}$ \\
\hline Scopus & $\begin{array}{l}\text { (Alnasser, et } \\
\text { al., 2019) }\end{array}$ & Twason & $\begin{array}{c}\text { Este estudo teve como objetivo determinar a } \\
\text { viabilidade de um intervalo de perda de peso na } \\
\text { ação que testa a efetividade de TWazon, } \\
\text { originalmente um aplicativo árabe projetado } \\
\text { para perda de peso, que promove mudanças no } \\
\text { estilo de vida, especificamente para Populações } \\
\text { árabes. }\end{array}$ & $\begin{array}{l}\text { Demonstramos que de acordo com o TWazon app } \\
\text { renderiza positivas mudanças no peso corporal,IMC e } \\
\text { energy dentro de intervenções centrad as em aplicativos } \\
\text { tem o potencial para ser efetivo na promoção da perda } \\
\text { de peso , saúde e estilo de vida em populações } \\
\text { semelhantes na Arábia Saudita }\end{array}$ & \\
\hline Scopus & $\begin{array}{c}\text { (Alturki e } \\
\text { Gay, 2019) }\end{array}$ & ASKER & $\begin{array}{l}\text { Explorar a oportunidade e a necessidade de } \\
\text { desenvolver um app árabe sobre perda de peso } \\
\text { que forneça conteúdo localizado e resolva os } \\
\text { problemas dos aplicativos existentes }\end{array}$ & $\begin{array}{l}\text { A amostra que utilizou o app relatou que o design do } \\
\text { aplicativo é iterativo e os recursos motivacionais são } \\
\text { faceis de usar.Facilita a mudança de comportamento não } \\
\text { saudáveis no estilo de vida da comunidade saudita }\end{array}$ & $\begin{array}{c}\text { Para ter sucesso, o grupo alvo, as características } \\
\text { motivacionais e as normas sociais e da cultura } \\
\text { precisam ser considerados. }\end{array}$ \\
\hline
\end{tabular}




\begin{tabular}{|c|c|c|c|c|c|}
\hline Scopus & $\begin{array}{l}\text { (Boels, et } \\
\text { al., 2019) } \\
\text { Noruega }\end{array}$ & Trigger & $\begin{array}{c}\text { Nosso objetivo é avaliar a eficácia de educação } \\
\text { sobre autogestão do diabetes por meio de um } \\
\text { aplicativo para smartphone em pacientes com } \\
\text { Diabetes tipo } 2 \\
\end{array}$ & $\begin{array}{l}\text { O teste fornecerá evidencias sobre a eficácia de um } \\
\text { aplicativo para smatphone recém -desenvolvido para } \\
\text { acionar o autogerenciamento de diabetes tipo } 2\end{array}$ & \\
\hline Scopus & $\begin{array}{l}\text { (Gilliland, et } \\
\text { al., 2015) } \\
\text { Canadá }\end{array}$ & SmartAPPetite & $\begin{array}{l}\text { "SmartAPPetite": um aplicativo para } \\
\text { smartphone ou aplicativo, projetado para } \\
\text { incentivar uma alimentação saudável } \\
\text { produzindo barreiras educacionais, } \\
\text { comportamentais e econômicas e acesso a } \\
\text { comida saudável local. }\end{array}$ & $\begin{array}{c}\text { O estudo alcançou o objetivo de mudança de } \\
\text { comportamento através da criação de cadeias de } \\
\text { informações alimentares que orientaram os usuários com } \\
\text { dicas de alimentação, receitas que incorporam esses } \\
\text { alimentos e, finalmente,para fornecedores específicos, } \\
\text { que venderam esses alimentos, tornando saudáveis } \\
\text { 9escolhas alimentares mais visíveis e, portanto, mais } \\
\text { fáceis de fazer. Os participantes fizeram uso de } \\
\text { SmartAPPetite e o comportamento positivo foi auto- } \\
\text { relatado }\end{array}$ & $\begin{array}{l}\text { Deve, se possível demonstrar se a cutucada dos } \\
\text { participantes via o projeto SmartAPPetite tem } \\
\text { efeitos positivos mantidos e se a mudança } \\
\text { comportamental na alimentação saudável, }\end{array}$ \\
\hline $\begin{array}{l}\text { Web of } \\
\text { science }\end{array}$ & $\begin{array}{l}\text { (Timmers, et } \\
\text { al., 2019) } \\
\text { Holanda }\end{array}$ & Significently & $\begin{array}{l}\text { Determinar se educar pacientes com TKR com } \\
\text { tempo, pós-operatório de informações diárias } \\
\text { sobre cuidados por meio de um aplicativo } \\
\text { podem levar a uma diminuição de seus níveis de } \\
\text { dor comparada àqueles que apenas recebem } \\
\text { informações padrão sobre sua recuperação } \\
\text { através do aplicativo }\end{array}$ & $\begin{array}{l}\text { A ação e educação diária dos pacientes por meio do } \\
\text { aplicativo significently auxiliou na diminuição do nível } \\
\text { de dor e melhorou o funcionamento físico, qualidade de } \\
\text { vida, capacidade de realizar fisioterapia e exercícios de } \\
\text { autocuidado diário, satisfação com informação, } \\
\text { percepção do envolvimento hospitalar e o consumo de } \\
\text { assistência médica comparado à educação padrão do } \\
\text { paciente. }\end{array}$ & $\begin{array}{c}\text { Devido ao crescente número de pacientes com } \\
\text { TKR e a ênfase crescente no autogerenciamento, } \\
\text { é sugerível o uso de um aplicativo com pós- } \\
\text { operatório oportuno }\end{array}$ \\
\hline $\begin{array}{l}\text { Web of } \\
\text { science }\end{array}$ & $\begin{array}{l}\text { (Ogden, } \\
\text { Maxwell e } \\
\text { Wong, } \\
\text { 2019) } \\
\text { Reino Unido }\end{array}$ & Ladle & $\begin{array}{l}\text { Desenvolver um comportamento baseado em } \\
\text { evidências com o uso de aplicativo de baixo } \\
\text { custo para controle de peso }\end{array}$ & $\begin{array}{l}\text { Ladle oferece uma alternativa baseada em evidências a } \\
\text { mais intensivas intervenções face a face, podendo } \\
\text { melhorar os hábitos e é menos demorado e dispendioso } \\
\text { de entregar. }\end{array}$ & N/A \\
\hline $\begin{array}{l}\text { Web of } \\
\text { science }\end{array}$ & $\begin{array}{l}\text { (Hartin, et } \\
\text { al., 2016) } \\
\text { Reino Unido }\end{array}$ & Gray matters & $\begin{array}{l}\text { Reduzir o risco futuro de desenvolver doença de } \\
\text { Alzeimer, enquanto a curto prazo, promover a } \\
\text { saúde vascular, através da mudança de } \\
\text { comportamento }\end{array}$ & $\begin{array}{l}\text { A natureza do telefone celular se destacou como uma } \\
\text { plataforma de entrega para a intervenção, permitindo a } \\
\text { destinação de material de intervenção educacional, ao } \\
\text { mesmo tempo em que encoraja a mudança de } \\
\text { comportamento. }\end{array}$ & $\begin{array}{c}\text { Deve ser feito um esforço sustentado para manter } \\
\text { os comportamentos alcançados devem mitigar o } \\
\text { risco futuro de doença de alzeimer }\end{array}$ \\
\hline $\begin{array}{l}\text { Web of } \\
\text { science }\end{array}$ & $\begin{array}{l}\text { (Davies, et } \\
\text { al., 2015) } \\
\text { Austrália }\end{array}$ & Hep b story & $\begin{array}{c}\text { Desenvolver um app culturalmente apropriado } \\
\text { sobre a Hepatite B para os indígenas } \\
\text { australianos }\end{array}$ & $\begin{array}{c}\text { O aplicativo é culturalmente apropriado, áudiovisual, } \\
\text { interativo e os usuários podem escolher inglês ou Yolu } \\
\text { Matha (o idioma mais comum no leste Arnhem Land) } \\
\text { como idioma preferido. }\end{array}$ & Recomendamos o aplicativo para outros estudos \\
\hline
\end{tabular}


Research, Society and Development, v. 10, n. 13, e352101321334, 2021

(CC BY 4.0) | ISSN 2525-3409 | DOI: http://dx.doi.org/10.33448/rsd-v10i13.21334

\begin{tabular}{|c|c|c|c|c|c|}
\hline $\begin{array}{l}\text { Web of } \\
\text { science }\end{array}$ & $\begin{array}{l}\text { (Dalton, et } \\
\text { al., 2018) } \\
\text { Austrália }\end{array}$ & $\begin{array}{c}\text { Health and } \\
\text { Babies }\end{array}$ & $\begin{array}{l}\text { Analisar as características das participantes que } \\
\text { não concluíram os requisitos do estudo na } \\
\text { tentativa de identificar possíveis barreiras } \\
\text { associadas à implementação do aplicativo de } \\
\text { gravidez "Health and Babies". }\end{array}$ & $\begin{array}{l}\text { O App Health and Babies foi desenvolvido para fornecer } \\
\text { informação de saúde sobre gravidez precoce que } \\
\text { aumentariam a confiança materna e reduziriam a } \\
\text { ansiedade. Foram utilizadas estratégias de engajamento } \\
\text { para promover o uso. }\end{array}$ & N/A \\
\hline $\begin{array}{c}\text { Science } \\
\text { Direct }\end{array}$ & $\begin{array}{l}\text { (Hermanns, } \\
\text { et al., 2019) } \\
\text { Brasil }\end{array}$ & Flash & $\begin{array}{c}\text { Desenvolver e avaliar um programa estruturado } \\
\text { de educação e tratamento, denominado FLASH, } \\
\text { para ajudar os usuários do FSGM a entender e } \\
\text { usar as informações glicêmicas disponíveis para } \\
\text { otimizar o tratamento do diabetes. } \\
\end{array}$ & $\begin{array}{l}\text { O uso do FLASH pode levar para uma melhoria } \\
\text { significativa no controle glicêmico das pessoas com } \\
\text { diabetes em terapia intensiva com insulina. }\end{array}$ & N/A \\
\hline $\begin{array}{c}\text { Science } \\
\text { Direct }\end{array}$ & $\begin{array}{l}\text { (Ribeiro, et } \\
\text { al., 2016) } \\
\text { Portugal }\end{array}$ & Hарру & $\begin{array}{c}\text { Desenvolver um aplicativo para smartphone, } \\
\text { capaz de induzir mudanças de comportamento } \\
\text { em indivíduos }\end{array}$ & $\begin{array}{l}\text { O APP é simples e fácil de usar e pode ser uma } \\
\text { ferramenta eficaz para persuadir os usuários a mudar seu } \\
\text { comportamento em relação à prevenção do câncer. }\end{array}$ & $\begin{array}{l}\text { Serão necessários aprimoramentos no aplicativo } \\
\text { para continuar a envolver os usuários com o } \\
\text { Happy. Isso incluirá a adição de duas novas } \\
\text { funcionalidades: desafios e sociais. }\end{array}$ \\
\hline
\end{tabular}

Fonte: Autores (2020) 
No que se refere a categoria de interesse do aplicativo tivemos como principais assuntos: diabetes com quatro aplicativos, atividade física também com quatro aplicativos e perda de peso/obesidade, com três aplicativos; os demais aplicativo versaram sobre outras áreas, como pode ser visto no Quadro 2.

Quadro 2 - Categoria dos Aplicativos.

\begin{tabular}{|c|c|}
\hline Nome do Aplicativo & Categoria \\
\hline Grávida + & \multirow{4}{*}{ Diabetes } \\
\hline PregnantWithDiabets & \\
\hline Trigger & \\
\hline Flash & \\
\hline DiaCert & \multirow{4}{*}{ Atividade Física } \\
\hline YORwalK & \\
\hline VELT & \\
\hline Accupedo-Pro Pedometer & \\
\hline TWASON & \multirow{3}{*}{ Perda de Peso/Obesidade } \\
\hline ASKER & \\
\hline LADLE & \\
\hline Yagmap & \multirow{2}{*}{ Câncer } \\
\hline Happy & \\
\hline Recover-E & \multirow{2}{*}{ Pós Operatório } \\
\hline Significe NTLY & \\
\hline Grávida + & \multirow{2}{*}{ Gestação } \\
\hline Health and Babies & \\
\hline CarrotRewards & \multirow{12}{*}{ Categorias variadas } \\
\hline The Bridge & \\
\hline CATA mApp & \\
\hline Gray Matters & \\
\hline SmartPetite & \\
\hline Heart MappEducation & \\
\hline PneumoRischio & \\
\hline Pro Fibro & \\
\hline Thrive & \\
\hline MeKidney & \\
\hline Farmalarm & \\
\hline Hep B NTLY & \\
\hline
\end{tabular}

Fonte: Autores (2020).

Quanto às categorias qualitativas analisadas, utilizou-se os objetivos do artigo, desfechos e desdobramentos. Embora o objetivo principal dos artigos escolhidos tenha sido o desenvolvimento de um aplicativo específico e a educação que esse aplicativo para smartphone pudesse proporcionar a uma determinado grupo de indivíduos escolhidos, algumas nuances 
específicas apareceram: desenvolvimento e implementação do aplicativo; coleta do feedback do usuário sobre o aplicativo; avaliação do efeito do aplicativo sobre o público alvo; avaliação do aplicativo para capacitação do público alvo; exploração do impacto do aplicativo; exploração, desenvolvimento e aplicabilidade do aplicativo; avaliação da eficácia do aplicativo. No que se refere aos trabalhos analisados, tratamos as propostas de melhoria das tecnologias de "Desdobramento", e a conclusão das pesquisas de "desfecho"; porém, nem todos os trabalhos apresentaram desfecho e desdobramento, como pode ser visto no Gráfico 1.

Gráfico 1: Número de artigos que apresentam desfecho e desdobramento

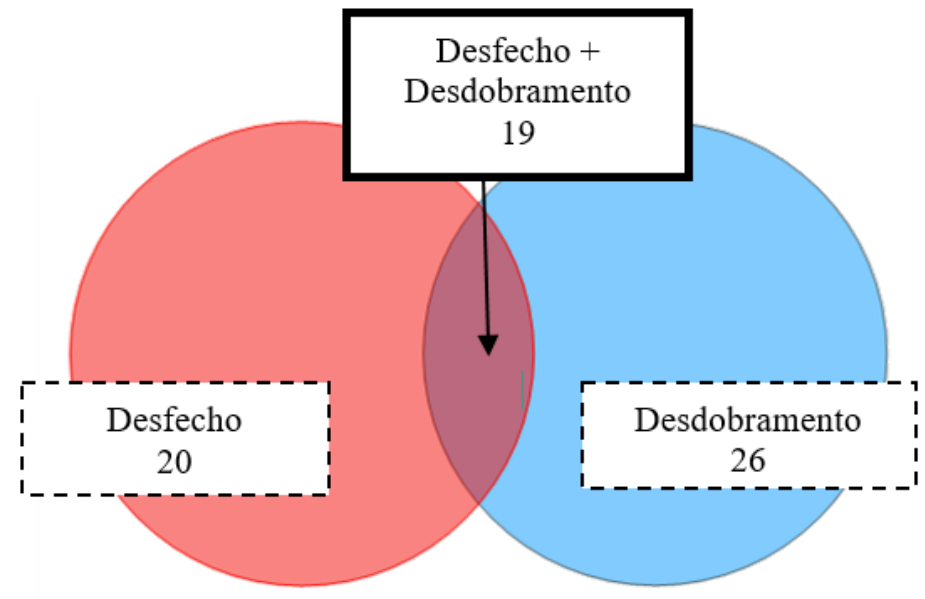

Fonte: Autores (2020)

\section{Discussão}

Os dados analisados revelam o ano de 2019 como o ano que mais apresentou publicação voltada para a Educação em Saúde para adultos. O estudo de Chaves, et al., (2018), indicava que ainda em 2018 haveria um acréscimo de 20\% em publicações voltadas para o uso de aplicativos móveis na Educação em Saúde e que os estudos nesta área aspiravam grande avanço nos anos seguintes. Os números de aplicativos em saúde estão aumentando e se tornando ferramentas muito úteis nos cuidados à saúde, o que pode ter se dado devido à popularização dos smartphones entre as pessoas, sendo essa uma oportunidade para que mais aplicativos sejam criados, principalmente (Machado, Turrini \& Sousa, 2020).

Dentre os países que mais publicam artigos na área, o Brasil desponta como um potencial expressivo, analisando-se os dados da Agência Nacional de Telecomunicações (ANATEL) que mostraram que o Brasil terminou o ano de 2017 com 242,1 milhões de aparelhos celulares e com a maior média de uso de smartphones do mundo (4 horas e 48 minutos). Esses dados indicam um caminho para onde o setor saúde deve se voltar e investir, com o objetivo de alcançar a nova geração de pacientes e para prestar serviços melhores e mais relevantes (Portal Saúde Business, 2017).

Em unanimidade, a língua dos artigos foi o Inglês; essa é uma tendência observada por alguns outros estudos, que justificam esse dado devido à uma soma de esforços no decorrer dos anos para aumentar a visibilidade dos artigos, dado o fato de que o inglês se tornou a língua de disseminação de novos conhecimentos científicos (Fuza, 2017; Nassi-Calò, 2016).

A análise da pesquisa mostrou como principais assuntos de interesse para desenvolvimento de aplicativos o diabetes, a atividade física e a perda de peso, corroborando com o estudo de Chaves, et al., (2018), onde patologias como diabetes, aparecem como interesse principal para monitoramento e seguimento ambulatorial e em domicílio para melhorias do cuidado em saúde da população, além de assuntos voltados para a gravidez e outras patologias. 
O leque para desenvolvimento de aplicativos móveis na área da Educação em Saúde tendo o paciente como usuário final, é vasto e abre uma gama de infinitas possibilidades para controle fora das unidades hospitalares e longe do alcance dos profissionais da saúde. Ainda, a possibilidade de controlar remotamente o paciente e sua patologia e do empoderamento dos pacientes no controle de suas doenças e participação no processo de melhoria e cura, só aumentam as vantagens da utilização desse aplicativos em celulares móveis. O uso de aplicativo móvel como ferramenta tecnológica educativa é um recurso promissor, pois tem aplicabilidade efetiva, amplo acesso, acessibilidade e visibilidade (Nicolau, Rocha e Bandeira, 2019; Neves, et al., 2016).

Pesquisas identificaram vários fatores considerados críticos para o sucesso de processos de melhoria contínua. O comprometimento da gerência é um dos principais fatores (Bessant, 2003), pois o trabalho gerencial por meio de atividades diárias promove a estabelecimento de uma cultura de aprendizado e inovação contínua. $\mathrm{O}$ uso de práticas, ferramentas e/ou técnicas permite o desenvolvimento de melhoria contínua.

Ao se debruçar sobre os números do gráfico 2, consta-se a presença de dois países emergentes, a Arábia Saudita e o Brasil. No que se refere à Arábia Saudita, 68 posição entre os países do mundo índice de inovação global (WIPO, 2019), evidencia-se um movimento que volta o país para uma perspectiva tecnológica para os próximos anos. Com a diminuição do uso dos recursos naturais, devido ao grande impacto positivo sobre a economia e criando oportunidades. É isso que a Arábia Saudita visa através da Visão 2030, que enfatizou o papel da inovação e pequenos projetos no desenvolvimento da economia, longe da dependência do petróleo. Esse reposicionamento corrobora com os dados desse trabalho que evidencia a Arábia Saudita entre países que não apenas inovam, como também produzem pesquisa sobre a temática. A maioria dos principais clusters (agrupamentos) de ciência e tecnologia encontra-se nos EUA, na China e na Alemanha, mas o Brasil também figura entre os 100 primeiros da lista (WIPO, 2019). Esse índice corrobora com os resultados do presente artigo que apresenta o número de desdobramentos das tecnologias analisadas.

\section{Conclusão}

Apresentamos neste estudo aplicativos móveis para smartphones voltados para a Educação em Saúde desenvolvidos no mundo nos últimos dez anos. A maior parte deles foi desenvolvido no ano de 2019 e países do Reino Unido, Arábia Saudita, Estados Unidos foram os que mais produziram aplicativos com essa finalidade. Além do objetivo de desenvolver o aplicativo, tais trabalhos buscavam a eficiência e efetividade na interatividade do usuário com o aplicativo. Desfechos e desdobramentos futuros, apresentados ao final dos artigos vislumbraram continuidade dos estudos com a melhoria e melhor utilização pelo usuário final.

A partir do que foi colhido como informação, pode ser visto que a inovação tecnológica é de grande ajuda quando o assunto é Educação em Saúde; o investimento no desenvolvimento de aplicativos com esse objetivo é promissor, ao se considerar o engajamento que as tecnologias têm nos dias atuais.

O leque de oportunidades para o desenvolvimento de aplicativos móveis para a Educação em Saúde dos detentores desses aparelhos é imenso, podendo levar a uma melhoria da qualidade da saúde aos portadores de diversas patologias no mundo e no Brasil e a possibilidade de uma melhor efetividade no que se refere ao empoderamento do paciente frente as condições de saúde que se não observadas poderão engrossar a procura pelos serviços de saúde.

Acredita-se que os aplicativos são oportunos para gerar educação e que o uso dessa tecnologia inovadora permita o desenvolvimento de melhoria contínua na saúde do público-alvo. Assim, ressalta-se a importância de que seja incentivada a criação de aplicativos móveis em benefício da saúde, baseados em evidência científica, para gerar educação e promoção em saúde de forma a melhorar a saúde dos usuários e dar melhor qualidade de vida aos mesmos. 


\section{Referências}

Abdulla, D. (2012). Attitudes of college students enrolled in 2-year health care programs towards online learning. Computers \& Education, 59(4), 1215-1223. https://doi.org/10.1016/j.compedu.2012.06.006

Abrams, T. E., Lloyd, A. A., Elzey, L. E., \& Hickerson, W. L. (2019). The Bridge: A mobile application for burn patients. Burns, 45(3), 699-704. https://doi.org/10.1016/j.burns.2018.09.028

Alnasser, A., Kyle, J., Aloumi, N., Al-Khalifa, A., \& Marais, D. (2019). The Twazon Arabic Weight Loss App: App-Based Intervention for Saudi Women With Obesity. JMIR mHealth and uHealth, 7(5), Artigo e10923. https://doi.org/10.2196/10923

Alturki, R., \& Gay, V. (2019). The Development of an Arabic Weight-Loss App Akser Waznk: Qualitative Results. JMIR Formative Research, 3(1), Artigo e11785. https://doi.org/10.2196/11785

Athilingam, P., Osorio, R. E., Kaplan, H., Oliver, D., O’neachtain, T., \& Rogal, P. J. (2016). Embedding Patient Education in Mobile Platform for Patients With Heart Failure. CIN: Computers, Informatics, Nursing, 34(2), 92-98. https://doi.org/10.1097/cin.0000000000000216

Bender, M. S., Martinez, S., \& Kennedy, C. (2015). Designing a Culturally Appropriate Visually Enhanced Low-Text Mobile Health App Promoting Physical Activity for Latinos. Journal of Transcultural Nursing, 27(4), 420-428. https://doi.org/10.1177/1043659614565249

Berrett, B., Murphy, J., \& Sullivan, J. (2015). Administrator Insights and Reflections: Technology Integration in Schools. The Qualitative Report. https://doi.org/10.46743/2160-3715/2012.1815

Bessant, J. (2003). Challenges in Innovation Management. In The International Handbook on Innovation (pp. 761-774). Elsevier. https://doi.org/10.1016/b978-008044198-6/50052-8

Boels, A. M., Vos, R. C., Dijkhorst-Oei, L.-T., \& Rutten, G. E. H. M. (2019). Effectiveness of diabetes self-management education and support via a smartphone application in insulin-treated patients with type 2 diabetes: results of a randomized controlled trial (TRIGGER study). BMJ Open Diabetes Research \& Care, 7(1), Artigo e000981. https://doi.org/10.1136/bmjdrc-2019-000981

Bonn, S. E., Alexandrou, C., Hjörleifsdottir Steiner, K., Wiklander, K., Östenson, C.-G., Löf, M., \& Trolle Lagerros, Y. (2018). App-technology to increase physical activity among patients with diabetes type 2 - the DiaCert-study, a randomized controlled trial. BMC Public Health, 18(1). https://doi.org/10.1186/s12889-018-5026-4

Borgen, I., Småstuen, M. C., Jacobsen, A. F., Garnweidner-Holme, L. M., Fayyad, S., Noll, J., \& Lukasse, M. (2019). Effect of the Pregnant+ smartphone application in women with gestational diabetes mellitus: a randomised controlled trial in Norway. BMJ Open, 9(11), Artigo e030884. https://doi.org/10.1136/bmjopen-2019-030884

Brett, J., Boulton, M., \& Watson, E. (2018). Development of an e-health app to support women prescribed adjuvant endocrine therapy after treatment for breast cancer. Patient Preference and Adherence, Volume 12, 2639-2647. https://doi.org/10.2147/ppa.s187692

Chow, M., Herold, D. K., Choo, T.-M., \& Chan, K. (2012). Extending the technology acceptance model to explore the intention to use Second Life for enhancing healthcare education. Computers \& Education, 59(4), 1136-1144. https://doi.org/10.1016/j.compedu.2012.05.011

Dale, L. P., White, L., Mitchell, M., \& Faulkner, G. (2019). Smartphone app uses loyalty point incentives and push notifications to encourage influenza vaccine uptake. Vaccine, 37(32), 4594-4600. https://doi.org/10.1016/j.vaccine.2018.04.018

Dalton, J. A., Rodger, D., Wilmore, M., Humphreys, S., Skuse, A., Roberts, C. T., \& Clifton, V. L. (2018). The Health-e Babies App for antenatal education: Feasibility for socially disadvantaged women. PLOS ONE, 13(5), Artigo e0194337. https://doi.org/10.1371/journal.pone.0194337

Davies, J., Bukulatjpi, S., Sharma, S., Caldwell, L., Johnston, V., \& Davis, J. S. (2015). Development of a Culturally Appropriate Bilingual Electronic App About Hepatitis B for Indigenous Australians: Towards Shared Understandings. JMIR Research Protocols, 4(2), Artigo e70. https://doi.org/10.2196/resprot.4216

Doyle, N., Murphy, M., Brennan, L., Waugh, A., McCann, M., \& Mellotte, G. (2019). The "Mikidney" smartphone app pilot study: Empowering patients with Chronic Kidney Disease. Journal of Renal Care, 45(3), 133-140. https://doi.org/10.1111/jorc.12281

Dwivedi, A., Dwivedi, P., Bobek, S., \& Sternad Zabukovšek, S. (2019). Factors affecting students' engagement with online content in blended learning. Kybernetes, 48(7), 1500-1515. https://doi.org/10.1108/k-10-2018-0559

Oliveira, A. R. F., Alencar, M. S. M. (2017). O uso de aplicativos de saúde para dispositivos móveis como fontes de informação e Educação em Saúde. RDBCI: Revista Digital de Biblioteconomia e Ciência da Informação, 15(1), 234, 31 jan. https://doi.org/10.20396/rdbci.v0i0.8648137.

Fontana, R. T., Flores, F. R., Silva, K. C., Thomas, L. S., Pires, L. G., Oliveira, N. G., \& Feller, S. d. R. (2020). Reflexões sobre a Educação em Saúde como um processo emancipatório. Brazilian Journal of Health Review, 3(3), 5196-5203. https://doi.org/10.34119/bjhrv3n3-096

Fuza, Â. F. (2017). O papel da língua inglesa na publicação acadêmico-científica: reflexões teóricas e o caso dos cursos de escrita on-line brasileiros. Signótica, 29(2), 302. https://doi.org/10.5216/sig.v29i2.43926

Gilliland, J., Sadler, R., Clark, A., O’Connor, C., Milczarek, M., \& Doherty, S. (2015). Using a Smartphone Application to Promote Healthy Dietary Behaviours and Local Food Consumption. BioMed Research International, 2015, 1-11. https://doi.org/10.1155/2015/841368

Glynn, L. G., Hayes, P. S., Casey, M., Glynn, F., Alvarez-Iglesias, A., Newell, J., ÓLaighin, G., Heaney, D., O’Donnell, M., \& Murphy, A. W. (2014). Effectiveness of a smartphone application to promote physical activity in primary care: the SMART MOVE randomised controlled trial. British Journal of General Practice, 64(624), e384-e391. https://doi.org/10.3399/bjgp14x680461 
Hartin, P. J., Nugent, C. D., McClean, S. I., Cleland, I., Tschanz, J. T., Clark, C. J., \& Norton, M. C. (2016). The Empowering Role of Mobile Apps in Behavior Change Interventions: The Gray Matters Randomized Controlled Trial. JMIR MHealth and UHealth, 4(3), e93. https://doi.org/10.2196/mhealth.4878

Heffernan, K. J., Chang, S., Maclean, S. T., Callegari, E. T., Garland, S. M., Reavley, N. J., Varigos, G. A., \& Wark, J. D. (2016). Guidelines and Recommendations for Developing Interactive eHealth Apps for Complex Messaging in Health Promotion. JMIR MHealth and UHealth, 4(1), e4423. https://doi.org/10.2196/mhealth.4423

Hermanns, N., Ehrmann, D., Schipfer, M., Kröger, J., Haak, T., \& Kulzer, B. (2019). The impact of a structured education and treatment programme (FLASH) for people with diabetes using a flash sensor-based glucose monitoring system: Results of a randomized controlled trial. Diabetes Research and Clinical Practice, 150, 111-121. https://doi.org/10.1016/j.diabres.2019.03.003

Inan, F. A., \& Lowther, D. L. (2009). Factors affecting technology integration in K-12 classrooms: a path model. Educational Technology Research and Development, 58(2), 137-154. https://doi.org/10.1007/s11423-009-9132-y

Índice Global de Inovação 2019: Índia registra grandes avanços, enquanto Suíça, Suécia, EUA, Países Baixos e Reino Unido lideram a classificação. Protecionismo comercial põe em risco o futuro da inovação. WIPO 2020. https://www.wipo.int/export/sites/www/pressroom/pt/documents/pr_2019_834.pdf, em julho de 2020

Johnson, D. W., \& Johnson, R. T. (2009). An Educational Psychology Success Story: Social Interdependence Theory and Cooperative Learning. Educational Researcher, 38(5), 365-379. https://doi.org/10.3102/0013189x09339057

Machado, R. C. G., Turrini, R. N. T., \& Sousa, C. S. (2020). Mobile applications in surgical patient health education: an integrative review. Revista Da Escola de Enfermagem Da USP, 54. https://doi.org/10.1590/s1980-220x2018032803555

Martin, S., Diaz, G., Sancristobal, E., Gil, R., Castro, M., \& Peire, J. (2011). New technology trends in education: Seven years of forecasts and convergence. Computers \& Education, 57(3), 1893-1906. https://doi.org/10.1016/j.compedu.2011.04.003

Mendes, K. D. S., Silveira, R. C. de C. P., \& Galvão, C. M. (2008). Revisão integrativa: método de pesquisa para a incorporação de evidências na saúde e na enfermagem. Texto \& Contexto - Enfermagem, 17(4), 758-764. https://doi.org/10.1590/s0104-07072008000400018

Nassi-Calò, L. Estudo aponta que artigos publicados em inglês atraem mais citações. SciELO em Perspectiva, 2016 https://blog.scielo.org/blog/2016/11/04/estudo-aponta-que-artigos-publicados-em-ingles-atraem-mais-citacoes/

Neves, N. T. A. T., Araújo, Y. B., Costa, C. M., Cardoso, E. L. S., Ferreira, E. M. V. Tendências De Estudos Sobre Aplicativos Móveis Para Saúde: Revisão Integrativa. XV Congresso Brasileiro de Informática em Saude, p. 499-508, 2016.

Nicolau, L., Rocha, P., \& Bandeira, A. M. (2019). Uso de aplicativo móvel na promoção de saúde de pessoas com fissuras labiopalatinas: relato de experiência. Academus Revista Científica Da Saúde, 04(01), 14-21. https://doi.org/10.24118/reva1806.9495.4.1.2019.480

Nørgaard, S. K., Nichum, V. L., Barfred, C., Juul, H. M., Secher, A. L., Ringholm, L., Damm, P., Mathiesen, E. R. Use of the smartphone application "Pregnant with diabetes". Danish Medical Journal, 64(11), 1-5, 2017.

Ogden, J., Maxwell, H., \& Wong, A. (2019). Development and feasibility study of an app (Ladle) for weight loss and behaviour change. PeerJ, 7, e6907. https://doi.org/10.7717/peerj.6907

Oliveira, R. M. de, Duarte, A. F., Alves, D., \& Furegato, A. R. F. (2016). Development of the TabacoQuest app for computerization of data collection on smoking in psychiatric nursing. Revista Latino-Americana de Enfermagem, 24(0). https://doi.org/10.1590/1518-8345.0661.2726

Os impactos de aplicativos móveis no setor de Saúde. Portal Saúde Business. https://saudebusiness.com/ti-e-inovacao/os-impactos-de-aplicativos-moveis-nosetor-de-saude/\#: :text=\%C3\%89\%20bem $\% 20$ verdade $\% 20$ que $\% 20$ a,quando $\% 20 \mathrm{se} \% 20$ trata $\% 20 \mathrm{do} \% 20 \mathrm{Brasil}$.

Panatto, D., Domnich, A., Gasparini, R., Bonanni, P., Icardi, G., Amicizia, D., Arata, L., Bragazzi, N. L., Signori, A., Landa, P., Bechini, A., \& Boccalini, S. (2016). Development and preliminary data on the use of a mobile app specifically designed to increase community awareness of invasive pneumococcal disease and its prevention. Human Vaccines \& Immunotherapeutics, 12(4), 1080-1084. https://doi.org/10.1080/21645515.2015.1114196

Pivec, M. (2007). Editorial: Play and learn: potentials of game-based learning. British Journal of Educational Technology, 38(3), 387-393. https://doi.org/10.1111/j.1467-8535.2007.00722.x

Ragavan, M. I., Ferre, V., \& Bair-Merritt, M. (2019). Thrive: A Novel Health Education Mobile Application for Mothers Who Have Experienced Intimate Partner Violence. Health Promotion Practice, 21(2), 160-164. https://doi.org/10.1177/1524839919890870

Rahim Shilbayeh, S. A., Abutaily, S. A., Al Ghwairi, L. S., Al Madani, W. O., Almoussa, A. F., \& Alzahrani, S. A. (2019). Development And Testing Of An Educational Mobile Application For Improving Knowledge Among Saudi Patients Receiving Warfarin. International Research Journal of Pharmacy, 10(5), 213-221. https://doi.org/10.7897/2230-8407.1005192

Ribeiro, N., Moreira, L., Almeida, A. M., \& Santos-Silva, F. (2016). Happy: Cancer Prevention Using Smartphones. Procedia Computer Science, 100, 466473. https://doi.org/10.1016/j.procs.2016.09.183

Ribo, M., montiel, E., Sanjuan, E., Sanchis, M., Rubiera, M., Muchada, M., Boned, S., Juega, J., Rodriguez, N., Rodriguez-Luna, D., Pagola, J., \& Molina, C. (2017). Abstract TMP99: Farmalarm: App for Mobile Devices Improves Risk Factor Control After Stroke. Stroke, 48(suppl 1). https://doi.org/10.1161/str.48.suppl_1.tmp99

Shalan, A., Abdulrahman, A., Habli, I., Tew, G., Thompson, A. YORwalK: Desiging a smartphone exercise application for people with intermittent claudication. Studies in Health Technology and Informatics, 247, 311-315, 2018 
Research, Society and Development, v. 10, n. 13, e352101321334, 2021

(CC BY 4.0) | ISSN 2525-3409 | DOI: http://dx.doi.org/10.33448/rsd-v10i13.21334

Shukor, N. A., Tasir, Z., Van der Meijden, H., \& Harun, J. (2014). A Predictive Model to Evaluate Students' Cognitive Engagement in Online Learning. Procedia - Social and Behavioral Sciences, 116, 4844-4853. https://doi.org/10.1016/j.sbspro.2014.01.1036

Stauber, A., Schüßler, N., Palmdorf, S., Schürholz, N., Bruns, D., Osterbrink, J., \& Nestler, N. (2020). RECOVER-E - a mobile app for patients undergoing total knee or hip replacement: study protocol. BMC Musculoskeletal Disorders, 21(1). https://doi.org/10.1186/s12891-020-3090-2

Tibes, C. M. dos S., Dias, J. D., \& Zem-Mascarenhas, S. H. (2014). Aplicativos móveis desenvolvidos para a área da saúde no Brasil: revisão integrativa da literatura. Revista Mineira de Enfermagem, 18(2), 471-486. https://doi.org/10.5935/1415-2762.20140035

Timmers, T., Janssen, L., van der Weegen, W., Das, D., Marijnissen, W.-J., Hannink, G., van der Zwaard, B. C., Plat, A., Thomassen, B., Swen, J.-W., Kool, R. B., \& Lambers Heerspink, F. O. (2019). The Effect of an App for Day-to-Day Postoperative Care Education on Patients With Total Knee Replacement: Randomized Controlled Trial. JMIR MHealth and UHealth, 7(10), e15323. https://doi.org/10.2196/15323

Yuan, S. L. K., \& Marques, A. P. (2018). Development of ProFibro - a mobile application to promote self-care in patients with fibromyalgia. Physiotherapy, 104(3), 311-317. https://doi.org/10.1016/j.physio.2018.04.005 